OPEN ACCESS

Edited by:

Catherine Alix-Panabieres,

Centre Hospitalier Universitaire de

Montpellier, France

Reviewed by:

Talib Hassan Ali,

Thi Qar University, Iraq

Francesca Benedetti,

University of Maryland, United States

${ }^{*}$ Correspondence:

Mark Niedre

m.niedre@neu.edu

Specialty section:

This article was submitted to

Molecular and Cellular Oncology,

a section of the journal

Frontiers in Oncology

Received: 31 August 2020

Accepted: 14 October 2020

Published: 06 November 2020

Citation:

Williams AL, Fitzgerald JE, Ivich F, Sontag ED and Niedre M (2020) ShortTerm Circulating Tumor Cell Dynamics

in Mouse Xenograft Models and Implications for Liquid Biopsy.

Front. Oncol. 10:601085.

doi: 10.3389/fonc.2020.601085

\section{Short-Term Circulating Tumor Cell Dynamics in Mouse Xenograft Models and Implications for Liquid Biopsy}

\author{
Amber L. Williams ${ }^{1}$, Jessica E. Fitzgerald ${ }^{1}$, Fernando Ivich ${ }^{1}$, Eduardo D. Sontag ${ }^{1,2,3}$ \\ and Mark Niedre ${ }^{1 *}$ \\ 1 Department of Bioengineering, Northeastern University, Boston, MA, United States, ${ }^{2}$ Department of Electrical and \\ Computer Engineering, Northeastern University, Boston, MA, United States, ${ }^{3}$ Laboratory of Systems Pharmacology, \\ Harvard Medical School, Boston, MA, United States
}

Motivation: Circulating tumor cells (CTCs) are widely studied using liquid biopsy methods that analyze fractionally-small peripheral blood (PB) samples. However, little is known about natural fluctuations in CTC numbers that may occur over short timescales in vivo, and how these may affect detection and enumeration of rare CTCs from small blood samples.

Methods: We recently developed an optical instrument called "diffuse in vivo flow cytometry" (DiFC) that uniquely allows continuous, non-invasive counting of rare, green fluorescent protein expressing CTCs in large blood vessels in mice. Here, we used DiFC to study short-term changes in CTC numbers in multiple myeloma and Lewis lung carcinoma xenograft models. We analyzed CTC detections in over $100 \mathrm{~h}$ of DiFC data, and considered intervals corresponding to approximately $1 \%, 5 \%, 10 \%$, and $20 \%$ of the PB volume. In addition, we analyzed changes in CTC numbers over $24 \mathrm{~h}$ (diurnal) periods.

Results: For rare CTCs (fewer than 1 CTC per ml of blood), the use of short DiFC intervals (corresponding to small PB samples) frequently resulted in no detections. For more abundant CTCs, CTC numbers frequently varied by an order of magnitude or more over the time-scales considered. This variance in CTC detections far exceeded that expected by Poisson statistics or by instrument variability. Rather, the data were consistent with significant changes in mean numbers of CTCs on the timescales of minutes and hours.

Conclusions: The observed temporal changes can be explained by known properties of CTCs, namely, the continuous shedding of CTCs from tumors and the short half-life of CTCs in blood. It follows that the number of cells in a blood sample are strongly impacted by the timing of the draw. The issue is likely to be compounded for multicellular CTC clusters or specific CTC subtypes, which are even more rare than single CTCs. However, we show that enumeration can in principle be improved by averaging multiple samples, analysis of larger volumes, or development of methods for enumeration of CTCs directly in vivo.

Keywords: circulating tumor cells, dynamics, optical devices, fluorescence, liquid biopsy, flow cytometry 


\section{INTRODUCTION}

Circulating tumor cells (CTCs) are of great interest in cancer research because of their importance in hematogenous metastasis. CTCs shed from the primary tumor into the peripheral blood (PB), and a small fraction may form metastases. It is these metastases that are extremely difficult to control clinically and eventually result in the majority of cancer-related deaths $(1,2)$. Nearly all CTC clinical and pre-clinical research involves "liquid biopsy", wherein CTCs are isolated from fractionally small PB samples $(3,4)$. CTCs are extremely rare, and fewer than 1 CTC per $\mathrm{ml}$ of $\mathrm{PB}$ is associated with reduced overall survival for major cancers such as breast (5), colorectal (6), and prostate (7). Although there have been a number of large clinical studies in the last decade, the clinical value of CTC enumeration by liquid biopsy remains as yet unclear (8-10). One major challenge is CTC heterogeneity, which has driven major efforts toward development next-generation liquid biopsy methods that permit genotypic and phenotypic characterization of single CTCs $(11,12)$.

A less-studied problem is that of temporal heterogeneity of CTCs and PB sampling, by which we mean the short-term fluctuations in CTC numbers in $\mathrm{PB}$ that may be largely invisible to liquid biopsy. Liquid biopsy implicitly assumes that the number of CTCs in a blood sample is representative of the entire $\mathrm{PB}$ volume (PBV). Previous work has shown that this assumption may be statistically dubious in light of the rarity of CTCs and the fractionally small volume of samples $(13,14)$. With respect to the latter, in CellSearch (for example), 7.5-ml PB samples are analyzed which corresponds to about $0.015 \%$ of the $\sim 5 \mathrm{~L}$ human PBV (6). Other experimental microfluidic platforms analyze similarly small samples in the range of $2-10 \mathrm{ml}(0.004 \%-$ $0.02 \% \mathrm{PBV})(15-17)$. With respect to pre-clinical mouse studies $\mathrm{PB}$ collection is limited to $200 \mu \mathrm{l}$ every two weeks for nonterminal experiments (without fluid replacement). This is equivalent to about $10 \%$ of the $\sim 1.5$ - to 2 -ml mouse $\operatorname{PBV}(18,19)$.

The small number of previously-published theoretical treatments of this problem also assumes that CTC detection (sampling) statistics should follow a Poisson distribution $(13,20$, 21). This further implicitly assumes that CTCs are well-mixed in blood, and that the average number of CTCs in circulation does not change significantly over the minutes or hours surrounding the blood draw. However, there is relatively little experimental pre-clinical or clinical data to support these assumptions $(7,20$, 22, 23). The relative infrequency and small PBV of blood draws are major challenges. In small animal models, the rarity of CTCs in mouse models also means that the entire PBV often must be drawn and analyzed. Such terminal experiments preclude serial study in the same mouse. In summary, little is currently known about short-term fluctuations in CTC numbers in vivo.

"In vivo flow cytometry" (IVFC) is a general term for optical instrumentation designed to detect and enumerate circulating cells directly in vivo, most often using either fluorescence or photoacoustic contrast $(19,24)$. We recently developed "diffuse in vivo flow cytometry" (DiFC) specifically for enumeration of rare green fluorescent protein (GFP)-expressing CTCs in mouse models of metastasis (25-29). DIFC uses diffuse light to non-invasively and continuously interrogate PB flowing in large, deeply-seated vessels.
We recently used DiFC to monitor CTC dissemination in multiple myeloma (MM) and Lewis lung carcinoma (LLC) $(27,29)$ mouse xenograft models. We showed that DiFC permitted longitudinal study of CTCs and CTC multi-cellular clusters (CTCCs) in individual mice at burdens below 1 CTC per $\mathrm{ml}$ of PB.

In our previous work, we used DiFC to study only the timeaveraged (mean) number of CTCs in circulation on a given day, but did not consider the short-term dynamics of CTC detections over timescales of minutes or hours. These short-term variations are measured by DiFC and therefore potentially provide unique insights into temporal dynamics of CTCs in vivo. In this work, we analyzed more than $100 \mathrm{~h}$ of DiFC data taken in mice, and considered sample intervals equivalent to approximately $1 \%, 5 \%, 10 \%$, and $20 \%$ of the PBV. We analyzed how CTC numbers fluctuated over the timescales of minutes and hours. As we show, CTC numbers were far from steady state, and exhibited variability far exceeding that expected by Poisson statistics or DiFC operator variability. As we show, this suggests that CTCs are not well-mixed in PB in general, and that the mean number of CTCs may change significantly over relatively short timescales. This can be explained by the short half-life of CTCs in circulation coupled with intermittent shedding behavior of CTCs. It also follows that the number of cells in a blood sample and the subsequent CTC enumeration accuracy may be strongly impacted by the timing of the draw. This data is also generally consistent with the small number of previously published preclinical and clinical studies on this issue. However, our analysis also shows that accuracy may be markedly improved by analysis of larger blood volumes, averaging of multiple blood samples, or continuous in vivo monitoring.

\section{MATERIALS AND METHODS}

\section{DiFC Instrument and Signal Processing}

The DiFC instrument (Figure 1A) and data processing algorithms were described in detail previously $(26,27,29)$. Briefly, two specially designed fiber-optic probes (Figure 1B) are placed in-line along a major blood vessel (in this case, the ventral caudal bundle in the tail) as shown in Figure 1C. The probes have integrated lenses and filters that allow efficient fluorescent light collection and rejection of non-specific tissue autofluorescence. GFP-expressing CTCs are detected by laserinduced fluorescence as they pass through the DiFC field of view (Figure 1D, also see Supplementary Materials and Methods 1.1). DiFC therefore permits detection of moving CTCs in large blood vessels 1-2 mm deep in tissue. Analysis of peak amplitude, width, and order of detection between the two channels allows us to discriminate arterial from venous flow directions (26).

Detections of CTCs during a DiFC scan may be visualized using temporal raster plots, where each vertical black line represents a detection of a CTC (Figure 1E). Example DiFC data sets from our previous studies in an LLC sub-cutaneous (s.c.) flank tumor model (Figures 1F-H) and an MM disseminated xenograft model (Figures 1I-K) are shown $(27,29)$. As we discuss in more detail below, these two models provide complementary cases for analysis of CTC dynamics, namely, i) very rare CTCs disseminating from a single 


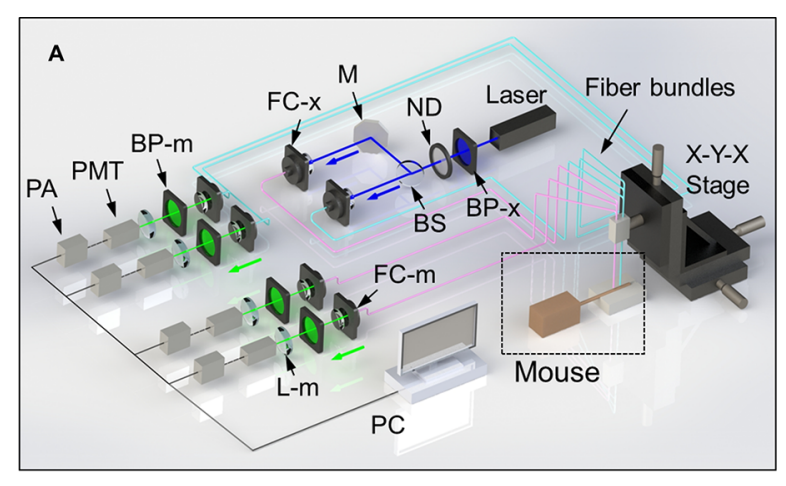

B

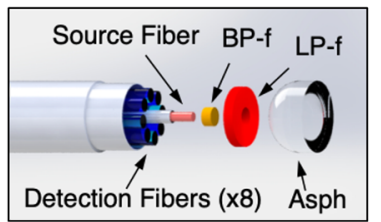

C
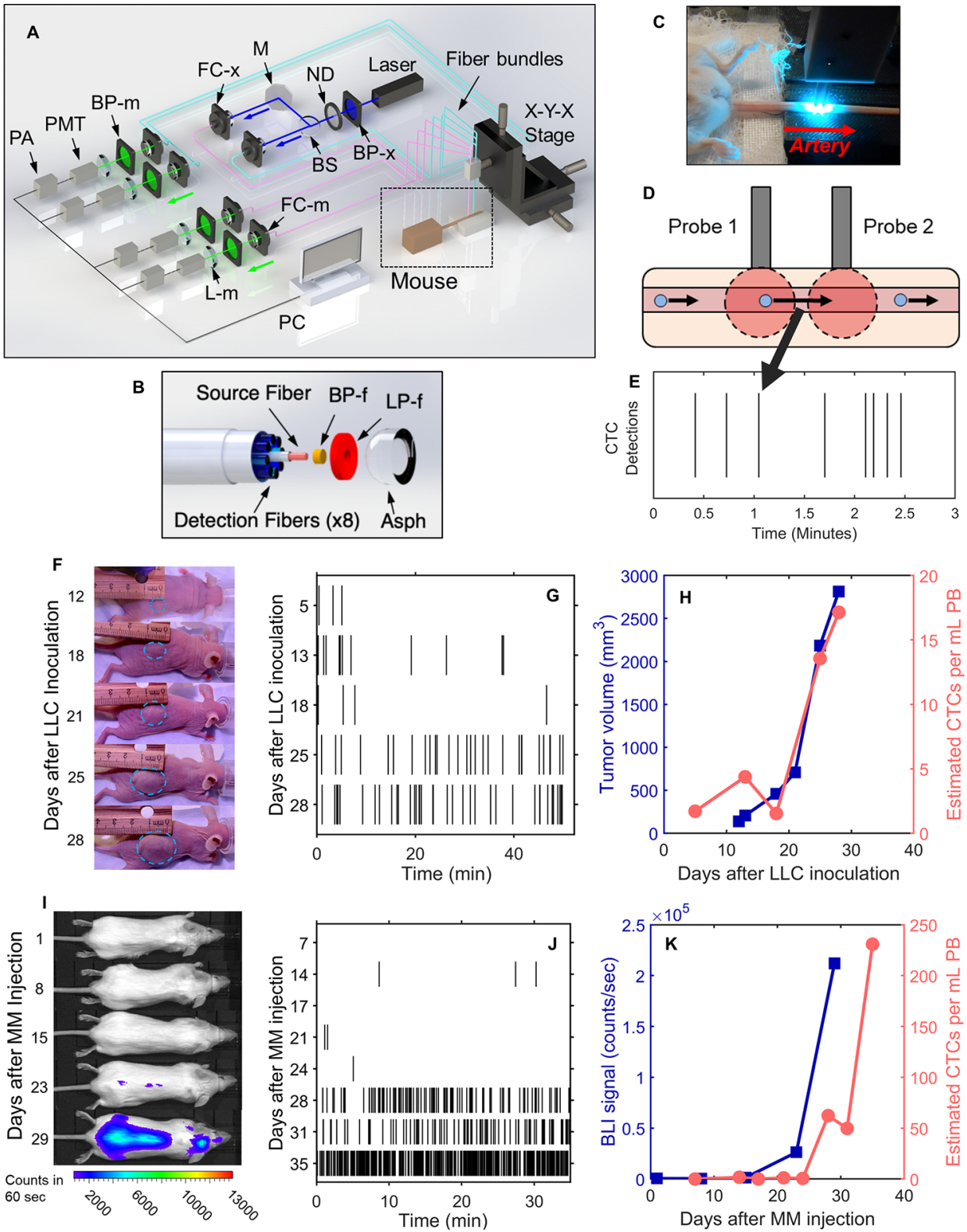

FIGURE 1 | DiFC allows non-invasive in vivo enumeration of rare circulating tumor cells (CTCs) in mice without drawing blood samples. (A) The DiFC instrument was described in detail previously $(27,29)$. The specially designed DiFC probes use two specially designed (B) optical fiber bundles that are placed on the (C) skin surface over the ventral side of the tail of a mouse. (D) When GFP-expressing CTCs pass through the DiFC field of view, the GFP is excited and the resulting fluorescent emission is detected by the probes. (E) The emission is identified as a CTC detection in time, which is visualized as a vertical black line on a raster plot. (F-H) A representative data set collected from an s.c. LLC-tumor bearing mouse. (F) Photographs of LLC tumor growth. (G) DiFC raster of CTC detections during tumor growth. (H) The tumor volume (blue squares) and mean rate of CTC detections (pink circles) over time. (I-K) Example MM-DXM mouse data. (I) Bioluminescence (BLI) images during MM growth. (J) DiFC raster plots of MM CTC detections. (K) BLI signal (blue squares) and mean rate of CTC detections (pink circles) over time. Panels (A, B) reproduced with permission from Patil et al. (27).

solid tumor (LLC) and, ii) more abundant CTCs disseminating from tumor distributed throughout the skeleton (MM).

We conservatively estimated that DiFC samples approximately $50 \mu \mathrm{l}$ of PB per minute (27). As such, DiFC allows sampling of the complete $\sim 1.5-2 \mathrm{ml}$ mouse PBV (potentially more than once) with single CTC sensitivity in a single, 30 - to 60 -min scan. Central to our analysis here is the assumption that the CTC count in a specific DiFC time interval is equivalent to the number of CTCs that would be counted in a blood sample of corresponding volume drawn from the same blood vessel at that time. For example, assuming that a mouse has $2 \mathrm{ml}$ of $\mathrm{PB}$, a $24 \mathrm{~s}$ DiFC scan interval is equivalent to approximately $20 \mu \mathrm{l}$ of blood, or about $1 \%$ of the PBV. We note 
this percentage is approximate and is intended to give a physical interpretation of DiFC scan intervals to the reader. Moreover, the percentage itself is not used in any calculations so that if the true PBV were slightly higher or lower than $2 \mathrm{ml}$ it would have no impact on results presented here. Mice used in each model were also approximately the same weight $(\sim 25 \mathrm{~g})$, so that this percentage would likewise be approximately consistent between mice. In this work, we considered all $24 \mathrm{~s}, 2 \mathrm{~min}, 4 \mathrm{~min}$ and $8 \mathrm{~min}$ DiFC scan intervals, equivalent to $20,100,200$, and $400 \mu \mathrm{l}$, or approximately $1 \%, 5 \%, 10 \%$, and $20 \%$ of the mouse PBV.

\section{DiFC Data Sets}

In this work, we analyzed five data sets as follows:

\section{Lewis Lung Carcinoma (LLC) Metastasis Model}

We re-analyzed previously reported DiFC data measured in s.c. Lewis lung carcinoma tumor bearing mice (29). In that study, we considered only the mean CTC numbers on each day but not shortterm fluctuations as we do here. Briefly, $10^{6}$ LLC cells expressing GFP (LL/2.GFP.Luc) cells were injected s.c. in the rear flank of 42 nude mice and allowed to grow for up to 3 weeks. DiFC was performed for 40- to 50-min, at least once per week. CTCs were observed in circulation as early as 5 days after inoculation, and there was a general increase in CTC detection rate with increasing primary tumor volume (Figures 1F-H). However, significant inter-mouse heterogeneity was observed in terms of both CTC numbers and lung metastasis formation. We used DiFC scans from this study where at least one CTC was detected ( $\mathrm{N}=102 \mathrm{DiFC}$ scans). CTC detection rates ranged from 0.019 to $1.05 \mathrm{CTCs}$ per $\mathrm{min}$, which is equivalent to 0.38 to $21.1 \mathrm{CTC}$ per $\mathrm{ml}$ of $\mathrm{PB}$ based on the $\mathrm{DiFC}$ sampling rate. We subsequently refer to these data as the "LLC dataset" below. A subset of 18 representative raster plots from this data set is shown in Supplementary Figure 1.

\section{Multiple Myeloma (MM) Disseminated Xenograft Model (DXM)}

We re-analyzed our previously reported DiFC data from an MM disseminated xenograft mouse model (DXM) (27). Briefly, $5 \times 10^{6}$ GFP-expressing MM.1S.GFP.Luc cells were injected intravenously (i.v.) via the tail vein of 10 SCID mice. After injection MM cells rapidly homed to the bone marrow niche, wherein they steadily proliferated and eventually re-entered circulation by the third week. DiFC was performed for 35 min twice weekly for up to 5 weeks. CTCs were relatively abundant (compared to the LLC model) and increased monotonically with bulk MM growth over time. Example data for an MM-DXM mouse is shown in Figures 1I-K. In the analysis here, we used data sets from this study where $\mathrm{DiFC}$ detection rates exceeded 0.5 CTCs per minute only $(\mathrm{N}=18)$, and ranged from 0.6 to 19.6 CTCs per minute, which is equivalent to 12 to 392 CTCs per $\mathrm{ml}$ of PB. We refer to this as the "MM 35-min dataset" below. The complete set of DiFC raster plots are shown in Supplementary Figure 2.

\section{Twenty-Four-Hour DiFC Measurements in MM-DXM Mice}

We also performed new experiments in MM-DXM mice. All mice were handled in accordance with Northeastern University's
Institutional Animal Care and Use Committee (IACUC) policies on animal care. Animal experiments were carried out under Northeastern University IACUC protocol \#15-0728R. All experiments and methods were performed with an approval from and in accordance with relevant guidelines and regulations of Northeastern University IACUC.

We performed i.v. injection of $5 \times 10^{6} \mathrm{MM}$.1S.GFP.Luc cells in six, 8-week-old male severe combined immunodeficient SCID/Bg mice (Charles River) as in the MM-DXM model above (27). We performed cycles of four, 50-min DiFC scans over a 24-h period, beginning 4 weeks after inoculation. Institutional mouse housing followed a 7 am-to-7 pm light, and 7 pm-to-7 am dark cycle. To minimize possible bias in the start time for each cycle, the first DiFC scan was performed at either 7 am $(\mathrm{N}=7$ data sets $)$ or $7 \mathrm{pm}(\mathrm{N}=7$ data sets). DiFC scans were performed by one of three human operators, and the first operator was also randomized. CTC detection rates for these data ranged from 0 to 42 CTCs per minute, equivalent to 0 to $840 \mathrm{CTCs}$ per $\mathrm{ml}$ of blood. We refer to these as the "MM 24-h dataset" below. The complete set of 24-h DiFC measurements is shown in Supplementary Figure 3.

Since 24-h measurements required three human operators performing four alignments (physical repositioning) of the DiFC probe on the mouse tail in 24 -h periods, we also measured the inherent inter- and intra-operator variability in DiFC measurements (for details see Supplementary Materials and Methods 1.2). These data are referred to as "1-operator-withreposition dataset" ( $\mathrm{N}=7$ data sets) and "2-operators-withreposition dataset" ( $\mathrm{N}=6$ data sets) below.

\section{Limb-Mimicking Optical Phantom With Fluorescent Microspheres}

We also used a limb-mimicking optical flow phantom model as we have previously (27). We used Dragon Green fluorescence level 5 (DG5) reference microspheres (Cat. DG06M, Bangs Laboratories, Inc., Fishers, IN) suspended in PBS at concentrations of 200 or 400 microspheres per $\mathrm{ml}$ pumped through the phantom at linear flow speeds of 15,30, or $60 \mathrm{~mm} / \mathrm{s}$. We performed DiFC at the six concentration-speed combinations in triplicate for $35 \mathrm{~min}$ in each case $(\mathrm{N}=18)$. Microsphere suspensions were first sonicated to prevent aggregation and clumping and then mixed well to produce as homogenous a suspension as possible. In principle, this should yield DiFC detections that follow Poisson distributions. More details on these experiments are provided in Supplementary Materials and Methods 1.3.

\section{Simulated DiFC Data}

We also simulated Poisson-distributed sequences of DiFC data in silico. We generated 54, 35-min data sets using custom written code in Matlab (The Mathworks Inc., Natick, MA) with mean rates of detection in the same range as those measured in MM DXM mice. To compare MM mouse data to processes that are not described by a single Poisson, we also generated 35-min Poisson simulations where the mean CTC detection rate increased by a factor of two halfway through the scan, i.e., Poisson detections with mean rate $\lambda_{1}$ in the first $17.5 \mathrm{~min}$ and $\lambda_{2}=2 \lambda_{1}$ in the second $17.5 \mathrm{~min}$. We generated 54 such data sets. We also simulated DiFC data sets of the sum of two concurrent 
(simultaneous or merged) 35-min, Poisson-distributed sequences of mean $\lambda_{1}$ and $\lambda_{2}=2 \lambda_{1}$. More details are provided in Supplementary Materials and Methods 1.4.

\section{RESULTS}

\section{For Rare CTCs Small Samples Frequently Resulted in No CTC Detections}

We re-analyzed previously reported DiFC data from an LLC s.c. flank metastasis model (29). In this model, CTCs were rare throughout tumor growth. Specifically, in 102 DiFC scans, CTC count rates corresponded to approximately 0.38 to 21.1 CTCs per $\mathrm{ml}$ of $\mathrm{PB}$. Representative DiFC raster plots illustrating the range of the data set are shown in Figures 2A-C. Eighteen additional example data sets are also shown in Supplementary Figure 1.

We considered the number of CTCs detected in 24-s, 2-min, 4min, and- 8 min intervals during the DiFC scans. As above, these intervals were equivalent to approximately $20,100,200$, and $400 \mu \mathrm{l}$ of $\mathrm{PB}$, or about $1 \%, 5 \%, 10 \%$, and $20 \%$ of the mouse $\mathrm{PBV}$. Histograms of the number of CTC detections (for the data shown in Figures 2A-C) are shown for $24 \mathrm{~s}$ (Figures 2D-F), $2 \mathrm{~min}$ (Figures 2G-I), $4 \mathrm{~min}$ (Figures 2J-L), and $8 \mathrm{~min}$ (Figures 2M-O) intervals, respectively. These data show that the probability that at least one CTC was detected in a small temporal sample was in general very low. For example, considering a $1 \%$ PBV sample size and 0.4 CTCs per mL, no CTCs were detected in 99\% of equivalent blood samples (intervals) over the entire scan. Even for relatively high CTC burdens (14 CTCs per ml; Figure 2C), 1\% PBV sample sizes yielded zero CTC detections 79\% of the time (Figure 2F). As would be expected, this probability improved significantly when larger time intervals (equivalent blood samples) were considered. For example, for a CTC burden of 14 CTCs per $\mathrm{ml}$ and an equivalent 20\% PBV sample (Figure 20), at least one CTC was detected $100 \%$ of the time.

Visualizing the data another way, the fraction of intervals for which at least 1 CTC was detected for all DiFC scans in the LLC dataset are shown in Figures 2P-S. In combination, these data underscore the fact that analysis of fractionally small blood samples (1\%-5\% PBV) frequently resulted in detection of no CTCs, even though CTCs were present in the blood in all cases. Hence these data provide direct experimental validation of the notion that "more blood is better" for detection of CTCs (14). Analysis of larger blood samples would further improve this: for example, analysis of $30 \%$ of the PBV would usually (> 50\% of possible PBV samples) result in detection of at least 1 CTC in half of the LLC data sets here.

\section{CTC Counts in Small Samples Were Generally Quantitively Inaccurate}

We also considered CTC enumeration accuracy from fractionally small blood samples. To study this, we used DiFC data measured in MM xenograft mice ("MM 35-min dataset"), (27) where CTCs were
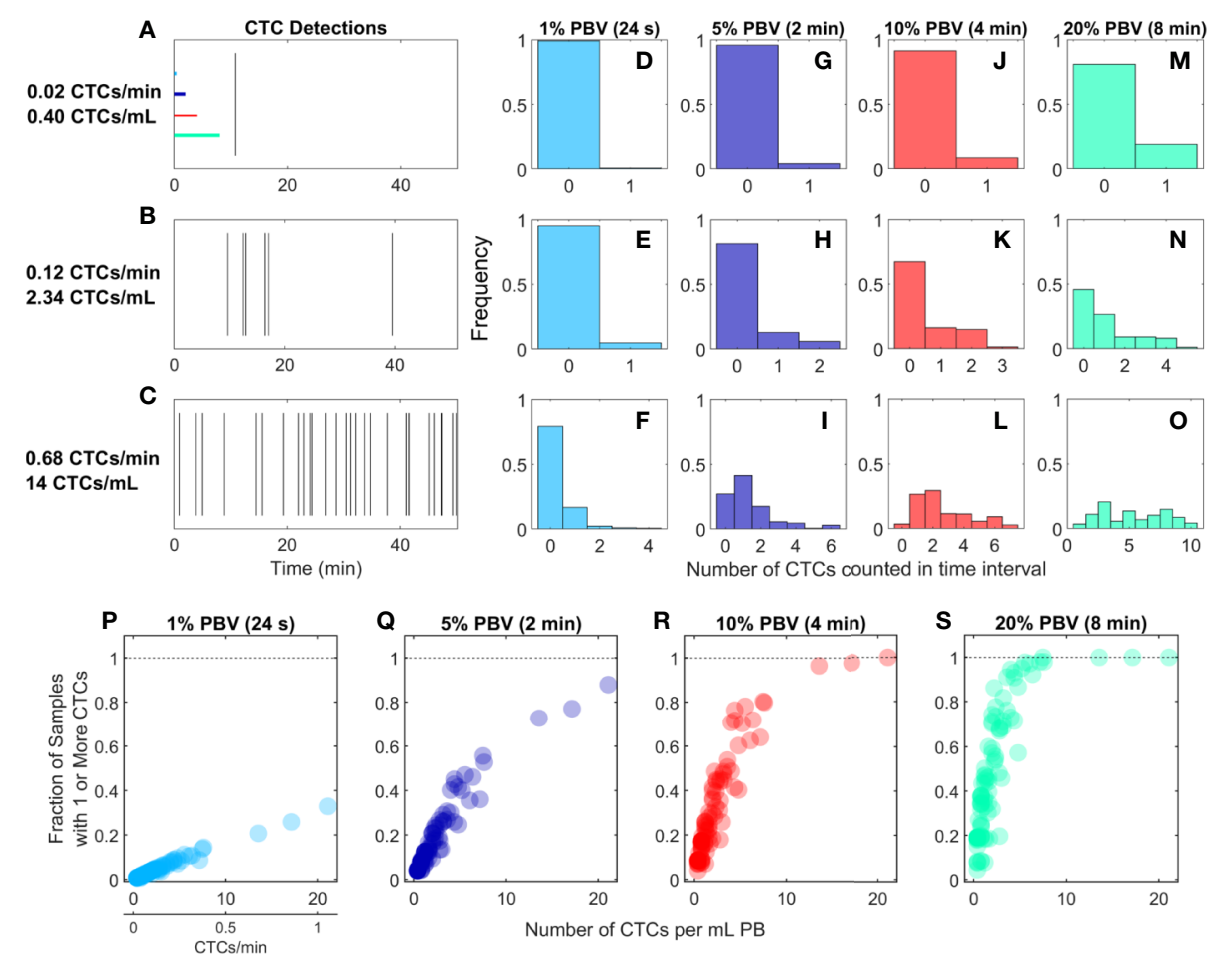

FIGURE 2 | For rare CTCs, small blood samples frequently resulted in no DiFC detections. (A-C) Representative DiFC raster plots of LLC-CTC detections for average count rates of (A) 0.4 , (B) 2.3, and (C) 14 CTCs per ml PB. Colored horizontal lines show the relative length of approximately $1 \%$ (24 s), 5\% (2 min), $10 \%$ (4 min), and 20\% (8 min) intervals (blood sample size). Distributions of CTC counts in detections intervals are shown for (D-F) 1\% PBV, (G-I) 5\%, (J-L) 10\%, and (M-O) 20\% PBV DiFC scan intervals. The fraction of intervals where 1 or more CTCs were detected for (P) 1\%, (Q) 5\%, (R) 10\%, and (S) 20\% PBV scan intervals. 
more abundant than in the LLC tumor model above. A representative 35-min DiFC raster plot from an MM xenograft mouse, measured 31 days after engraftment of MM cells is shown in Figure 3A. (The full set of $18 \mathrm{DiFC}$ scans from this data set is shown in Supplementary Figure 2.) The number of CTCs counted in sliding $~ 1 \%, 5 \%, 10 \%$, and 20\% PBV intervals are shown in Figures 3B-E, respectively. We also calculated the mean number of CTCs over the entire DiFC scan (black horizontal line in each case). By inspection, CTC counts varied significantly during the scan, with periods of relatively high and low detection rates.

These data illustrate the large range of CTC detection rates measured over 35-min scans. For example, considering a 5\% PBV interval (which is typical volume for a mouse blood collection experiment), equivalent detection rates ranged from 0 to 38 CTCs per sample. In other words, if PB was collected from this blood vessel, $100-\mu \mathrm{l}$ samples drawn at different times (separated by just a few minutes) would have yielded order-ofmagnitude or more differences in CTC numbers.

The histograms of these data (i.e., the number of CTC detections for all possible $1 \%, 5 \%, 10 \%$, and $20 \% \mathrm{PBV}$ intervals) are shown in Figures 3F-I, along with the mean number of CTCs detected over the full scan (vertical black lines). We compared the measured distributions to Poisson distributions which, as noted above, are frequently assumed for liquid biopsy of PB $(13,20,21)$. These are indicated by the black dotted curves in Figures 3F-I. By inspection, the DiFCmeasured distribution of count rates did not appear to follow the Poisson distribution, particularly for larger (2-8 min) equivalent blood samples. The implications of this observation are discussed in more detail below.

We also computed the "deviation from the scan mean" (DFSM) for each observation (blood sample):
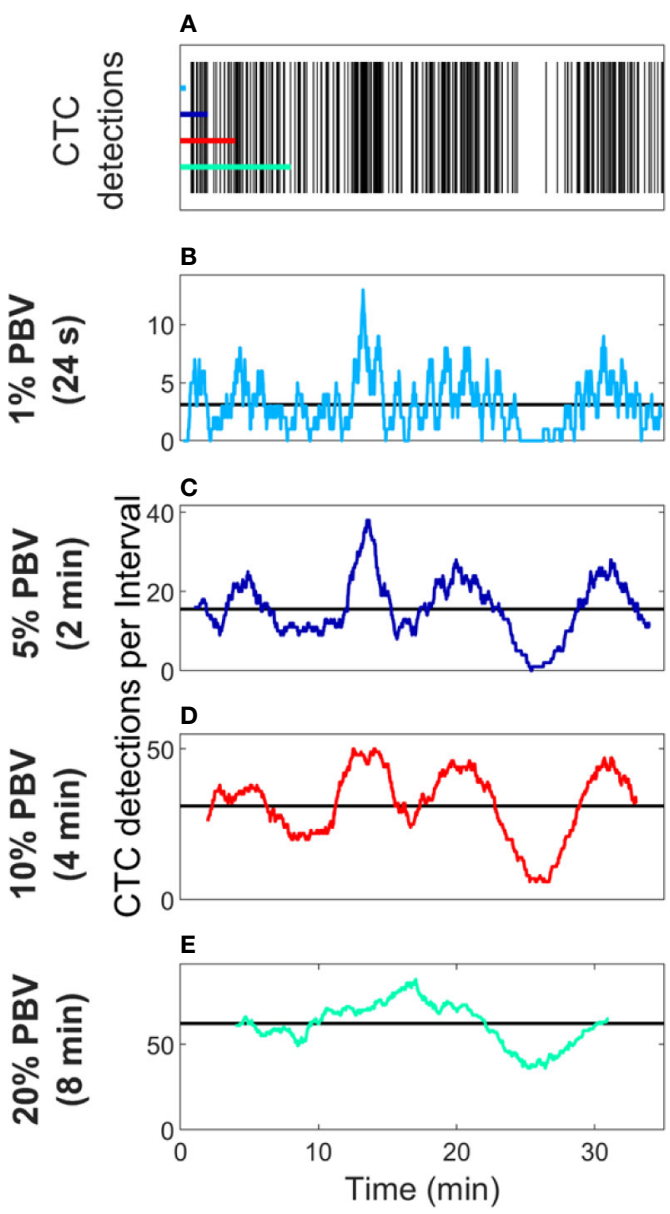
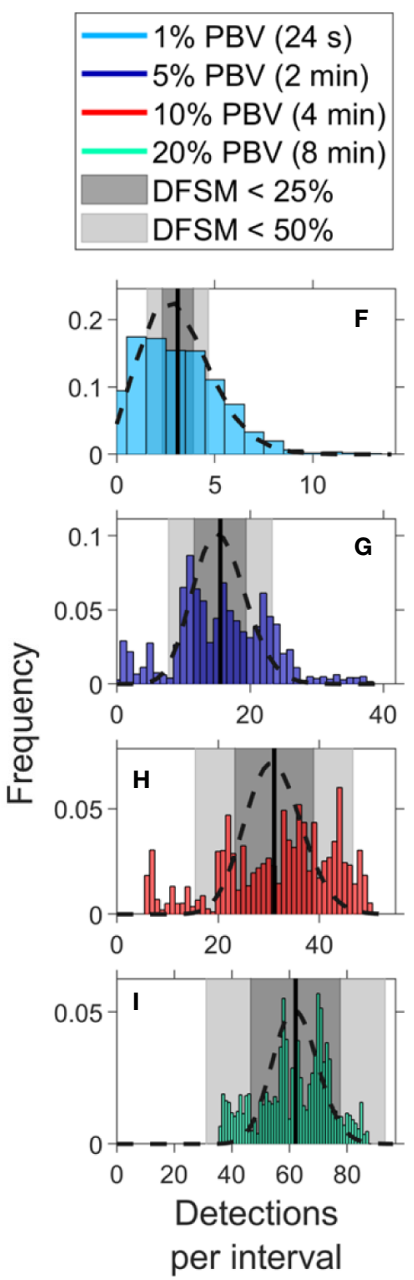

FIGURE 3 | Significant temporal fluctuation in CTC numbers were observed over the timescale of minutes. (A) Representative DiFC raster plot measured from a MM DXM bearing mouse. Colored horizontal lines show the length of each time interval (blood sample size). The number of CTCs counted in sliding (B) $1 \%$, (C) $5 \%$, (D) 10\%, and (E) 20\% equivalent PBV intervals are shown. Black horizontal lines identify the scan mean number of CTCs per interval. (F-I) The corresponding distributions of CTC counts for each equivalent PBV are shown. Black dashed lines indicate the distributions that would be expected from Poisson statistics. Black vertical lines show the mean number of CTCs. Gray shading denotes the range of DFSM $<25 \%$ (light gray) and < 50\% (dark gray). 


$$
\operatorname{DFSM}_{i}=\frac{\left|C_{i}-\lambda\right|}{\lambda} * 100 \%
$$

where $C_{i}$ is the number of CTCs in the $i^{\text {th }}$ equivalent sample and $\lambda$ is the mean number of CTC detections over the full scan for each sample size. The cumulative fraction of blood samples within 0-100\% DFSM for the data in Figures 3F-I are shown in Figure 4A. The cumulative fraction of samples for Poisson distributions of the same mean are also shown (dotted lines, same colors), again suggesting that experimental data diverged substantially from the Poisson behavior.

For example, these data can be interpreted as, "39\% of randomly selected 5\% PBV blood samples would yield a CTC count within $25 \%$ of the scan mean, whereas Poisson statistics predict that this number should be $69 \%$ ". It should also be noted that the large disagreement between expected Poisson behavior and measured behavior occurred in about half of the data sets. A representative plot from another DiFC scan where closer agreement was observed is shown in Figure 4B.

We next considered the fraction of intervals where the DFSM was equal to or less than $25 \%$ and $50 \%$ for all sample sizes in the complete "MM 35-min dataset". These data are summarized in
Figures 4C, D. Our use of 25\% and 50\% DFSM thresholds were selected since they are illustrative of sufficiently large error to affect prognostic classification-for example, in determining whether a sample has 4 or 5 CTCs (breast and prostate cancer) or 2 or 3 CTCs (colorectal cancer) (6-8).

Taken together, these data show that quantitative estimation of CTC numbers from single samples in mice is extremely challenging. For example, for 5\% PBV samples, the median probability for all scans of randomly obtaining a CTC count within $25 \%$ of the scan mean was only $41.9 \%$ (Figure 4C). Likewise, the probability of obtaining a CTC count within $50 \%$ of the mean was $72.1 \%$ (Figure 4D). All else being equal, use of larger blood sample volumes yielded higher probability of obtaining accurate count than smaller samples (14).

\section{There Were Significant Variations in CTC Detection Rates in 24-h Periods}

We also studied the 24-h variability in CTC detection rates in MM xenograft mice by performing four, 50 -min DiFC scans over 24 -h periods ("MM 24-h dataset"). Half of the data sets began at
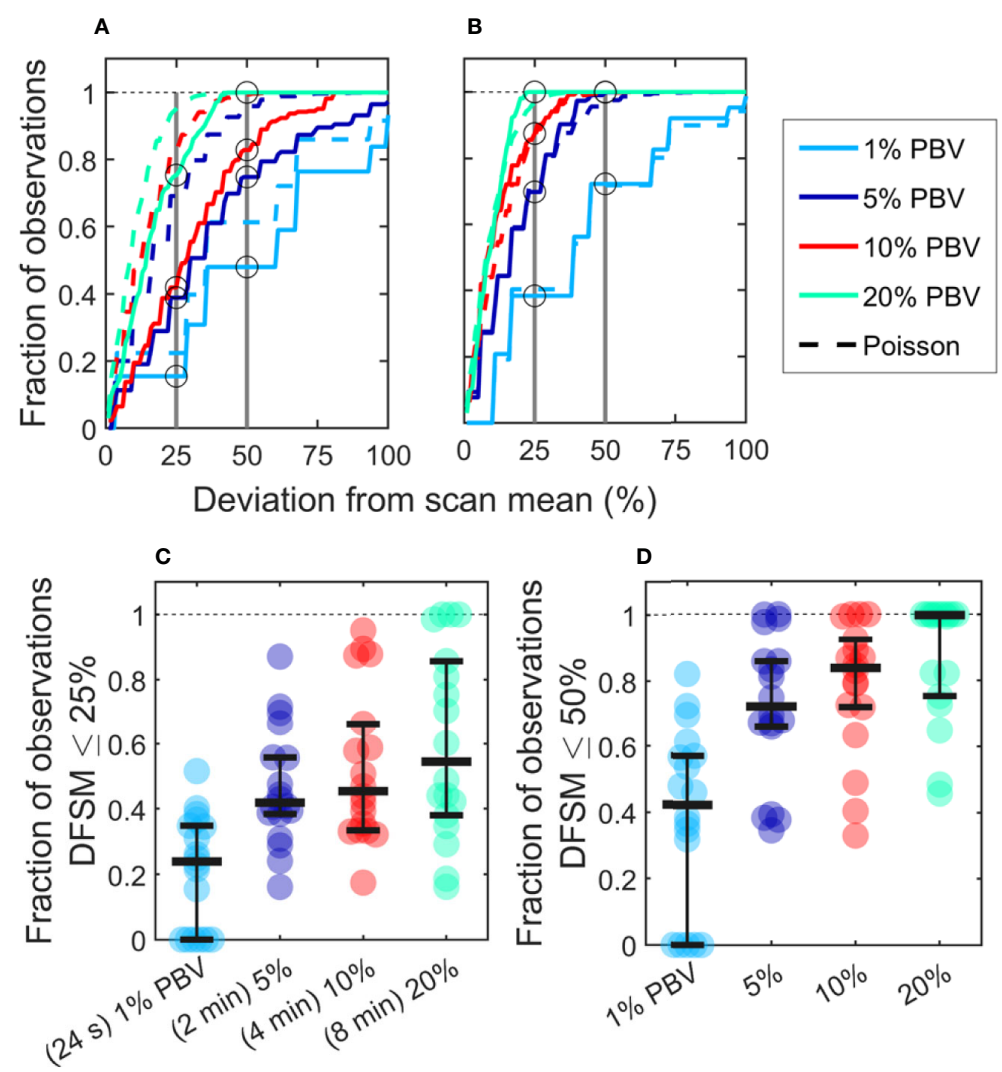

FIGURE 4 | CTC counts in small equivalent blood samples were generally quantitatively inaccurate. (A) Analysis of a representative DiFC data set measured in an MM xenograft mouse, showing the impact of the sample size on the percentage deviation of CTC counts in an interval from the scan mean (DFSM; see text for details). The fraction of observations within a given DFSM is shown in each case. Dotted lines indicate the expected distribution based on Poisson statistics. (B) A second example data set that more closely followed the expected Poisson behavior. (C) The fraction of equivalent blood samples with CTC counts within $25 \%$ of the scan mean (DFSM < 25\%) for all 18 DiFC scans in the MM 35-min dataset. (D) The fraction of samples within $50 \%$ of scan mean (DFSM < 50\%). Horizontal bars indicate the median, and first and third quartile for each blood sample size. 
7PM, and half began at 7 am to rule out the possibility that the start time could affect the measurements. In addition, the starting DiFC human operator (one of 3) was randomized. Two representative DiFC data sets from 24-h sessions are shown in Figures 5A-C. CTC detection rates in moving 2 min (5\% PBV) intervals are shown in Figures 5A, B. The 50-min average of each DiFC scan is also shown (dotted horizontal lines), as well as the average over the 24 -h period (solid horizontal lines). The mean count rates over the four DiFC scans for both mice are summarized for clarity in Figure 5C. As shown, the average measured detection rates changed by more than an order of magnitude depending on the time of day. The complete set of 14, 24-h DiFC scan sets from this data set are shown in Supplementary Figure 3.

To better quantify the 24 -h variability, the ratios of the maximum-to-minimum CTC detection rates for each scan are shown in the first column of Figure 5D. As shown, this varied from 2.5 to 152 . We note that we did not observe any specific circadian pattern to the data as others have reported for MM (23). Rather, the CTC count rates were seemingly randomly higher or lower at different times of days.

This 24-h variability was also greater than the fluctuations observed over a short timescale (as in Figure 3). To show this, DiFC scans from the "MM 35-min dataset" were each divided into the first 15 and last $15 \mathrm{~min}$ (separated by $5 \mathrm{~min}$ ), and the same maximum-to-minimum count rates ratios were calculated. These ranged from 1.05 to 4.4 (Figure 5D, column 2).

We note that 24-h measurements required up to three human operators performing four alignments (physical repositioning) of the $\mathrm{DiFC}$ probe on the mouse tail in each 24 -h dataset. To rule out the possibility that the observed variability in CTC detection rates (Figure 5C) was due to repositioning of the DiFC probe on the skin surface between scans (which could affect the collection efficiency of the system) and not biological fluctuations, we tested
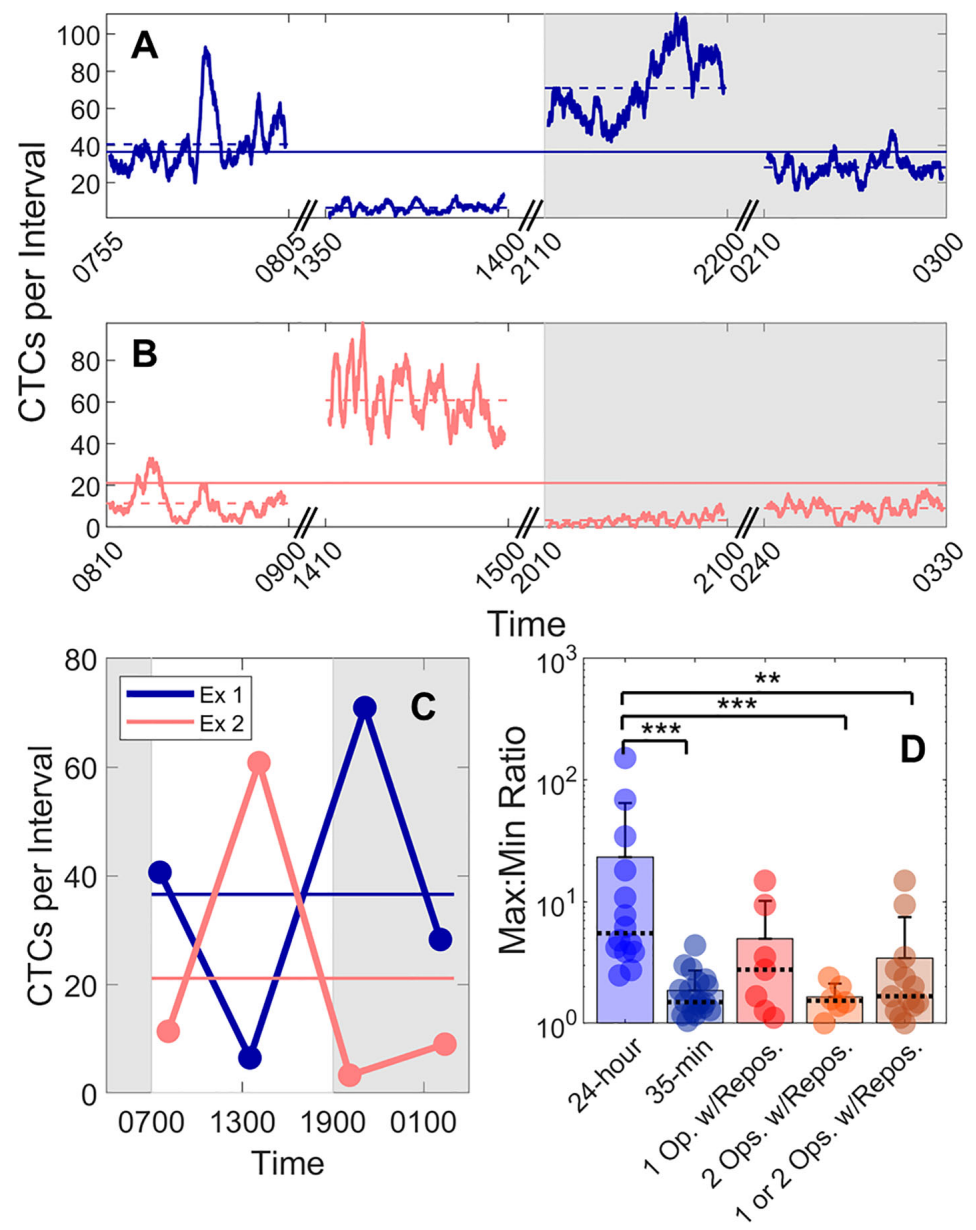

FIGURE 5 | Large fluctuations in CTC numbers were observed over 24-h cycles. (A, B) Example DiFC measurements taken over 24 h in two MM-bearing mice. The number of CTCs counted in 2 min (5\% PBV) sliding intervals is shown. The solid horizontal lines in each figure show the 24-h mean number of CTCs per 2 min interval, and the dashed horizontal lines show the local mean of the 50-min DiFC scan. Dark hours are shaded gray. (C) The mean DiFC count rates over the 24-h period are shown. (D) Ratios of maximum-to-minimum DiFC count rates are shown for MM 24-h, MM 35-min (no repositioning), 1-operator-with-reposition, and 2operators-with-reposition data sets. Bars represent the mean values with standard deviation error bars and dotted lines to identify the median values. Note the logarithmic y-axis. Kolmogorov-Smirnov test significance indicated by ** $(p$-value $<0.01)$ and ${ }^{\star \star \star}(p$-value $<0.001)$. 
the intra-operator reproducibility of DiFC count rates. To do this, we tested the intra- ("1-operator-with-reposition") and interoperator ("2-operators-with-reposition") reproducibility of DiFC count rates. The corresponding maximum-to-minimum DiFC count rate ratios ranged from 1.1 to 14.9 (Figure 5D column 3) and 1.0 to 2.4 (Figure 5D column 4), respectively.

Statistical analysis showed that the variability in mean DiFC count rate measured over $24 \mathrm{~h}$ was much larger than expected from either intra- or inter-operator repositioning variability. Specifically, when comparing $M M 24-h$ data $(\mathrm{N}=14)$ to $M M$ 35 -min $(\mathrm{N}=18)$, 1-operator-with-reposition $(\mathrm{N}=7)$, 2-operatorswith-reposition $(\mathrm{N}=6)$, and all reposition data $(\mathrm{N}=13)$ by a twosided, two-sample Kolmogorov-Smirnov test (MATLAB version 9.6), $\mathrm{p}$-values were $\mathrm{p}<0.001, \mathrm{p}=0.058, \mathrm{p}<0.001$, and $\mathrm{p}<0.01$, respectively. In addition, the intra- and inter-operator variability, as well as short-term fluctuations were not significantly different from each other (all $\mathrm{p}>0.15$ ).

\section{Variability in CTC Detection Rates In Vivo Was Higher Than Predicted by Poisson Statistics}

Because CTC detection is a random process, some inherent measurement variability is expected. However, as already noted the variability observed in DiFC data in vivo frequently exceeded that expected by Poisson statistics. To better study this, we plotted the variance of the CTC counts per interval against the mean. This was repeated for $24 \mathrm{~s}, 2 \mathrm{~min}, 4 \mathrm{~min}$, and $8 \mathrm{~min}$ intervals. Since the mean and variance of an ideal Poisson distribution are equal, if Poisson distributed CTC measurements should fall approximately along the 1:1 curve. However, as shown in Figures 6A-D, the measurement variance for the "MM 35-min dataset" was larger than the mean for about half the measurements. This deviation was significantly larger when considering the "MM 24-h dataset", where fluctuations were observed over 24-h periods (Figures 6E-H). Linear fits with a fixed intercept at zero resulted in slopes larger than 1 , demonstrating that the variance generally exceed the mean.

We note that in computing the mean and variances we analyzed all possible intervals in the DiFC scans (a "sliding window"). While this approach yielded significant overlap (nonindependence) between measurements, we showed explicitly that when large numbers of overlapping intervals are considered the variance converges to the non-overlapping case (see Supplementary Materials and Methods 1.5).

To rule out the possibility that this deviation from Poisson behavior was an artifact of DiFC measurement or data analysis methods, we repeated the analysis on in silico simulated data sets, where detections were Poisson distributed as shown in Figures 6I-L. We also performed DiFC measurements in a limb-mimicking optical flow phantom with suspensions of fluorescent microspheres that were well-mixed as shown in Figures 6M-P. In both cases, the variance more closely agreed with the scan mean, and linear fitting yielded slopes that were generally close to 1 . The slightly larger slopes observed in the phantom data likely resulted from microspheres settling in the syringe pump over the course of the DiFC scans, i.e., the spheres were not perfectly mixed.
The physical interpretation of these data is that the mean number of CTCs in the PB fluctuated significantly (i.e., was not in quasi-steady-state) over the time-scale of the DiFC experiments. To further test this, we simulated Poissondistributed data sets where the mean number of CTCs doubled halfway through the scan (Figures 6Q-T). These data more closely resemble the in vivo experimental data (Figures 6A-H). It is also worth noting that the in vivo DiFC data is not consistent with multiple concurrent Poisson processes. As shown in Figures 6U-X (and by the properties of Poisson statistics) this summation would exhibit Poisson behavior.

The implications of these data are discussed in more detail below. However, in practice, this means simply that the expected variability in CTC numbers in a single, randomly-drawn blood sample is actually much worse than would be expected by Poisson statistics as others have assumed $(13,20)$.

\section{Quantification of CTCs Can Be Improved by Averaging Multiple Samples}

Previous studies have shown that analysis of larger blood samples provides more accurate quantification of CTCs than smaller blood samples (14). The large temporal variability and deviation from Poisson behavior observed in our data further predicts that averaging multiple small blood samples over 24 -h periods should yield more quantitatively accurate estimates (versus the average) than a single, larger blood sample. As summarized in Figure 7, we considered a number of sample sizes and sample number combinations. We computed the number of samples where the DFSM $\leq 25 \%$ for each case. These are shown for (Figure 7A) $1 \%$, $2 \%$, two $1 \%, 4 \%$, four $1 \%, 20 \%, 80 \%$, and four $20 \%$ samples. Here, multiple samples were selected at least $6 \mathrm{~h}$ apart.

We then compared paired differences for equivalent total blood volumes taken continuously or throughout the 24 -h period as shown in Figure 7B. For example, we compared two, averaged 1\% PBV (24s) samples to a single 2\% PBV (2 min) measurement. Similarly, we compared four, $1 \%$ PBV samples (each taken at least $6 \mathrm{~h}$ apart) to a single $4 \%$ PBV sample, as well as four, $20 \% \mathrm{PBV}$ samples to one single $80 \%$ PBV sample (32 min). For nearly every data set, averaging multiple smaller blood samples yielded a higher likelihood of accurately estimating the $24-\mathrm{h}$ CTC rate compared to single, larger blood samples. This data demonstrates that while larger blood samples do improve the likelihood of accurately estimating CTC numbers, further improvements may be made by averaging smaller samples taken at different times of the day.

\section{DISCUSSION}

Although CTCs are widely studied using liquid biopsy, methods that involve analysis of single, fractionally-small $\mathrm{PB}$ samples inherently do not resolve temporal fluctuations in CTC numbers that can occur over the timescale of minutes or hours. As we have noted, DiFC samples large volumes of circulating PB continuously and non-invasively, and therefore provides unique insights into in vivo CTC dynamics in mouse models of metastasis. Our data in two xenograft models here showed that 

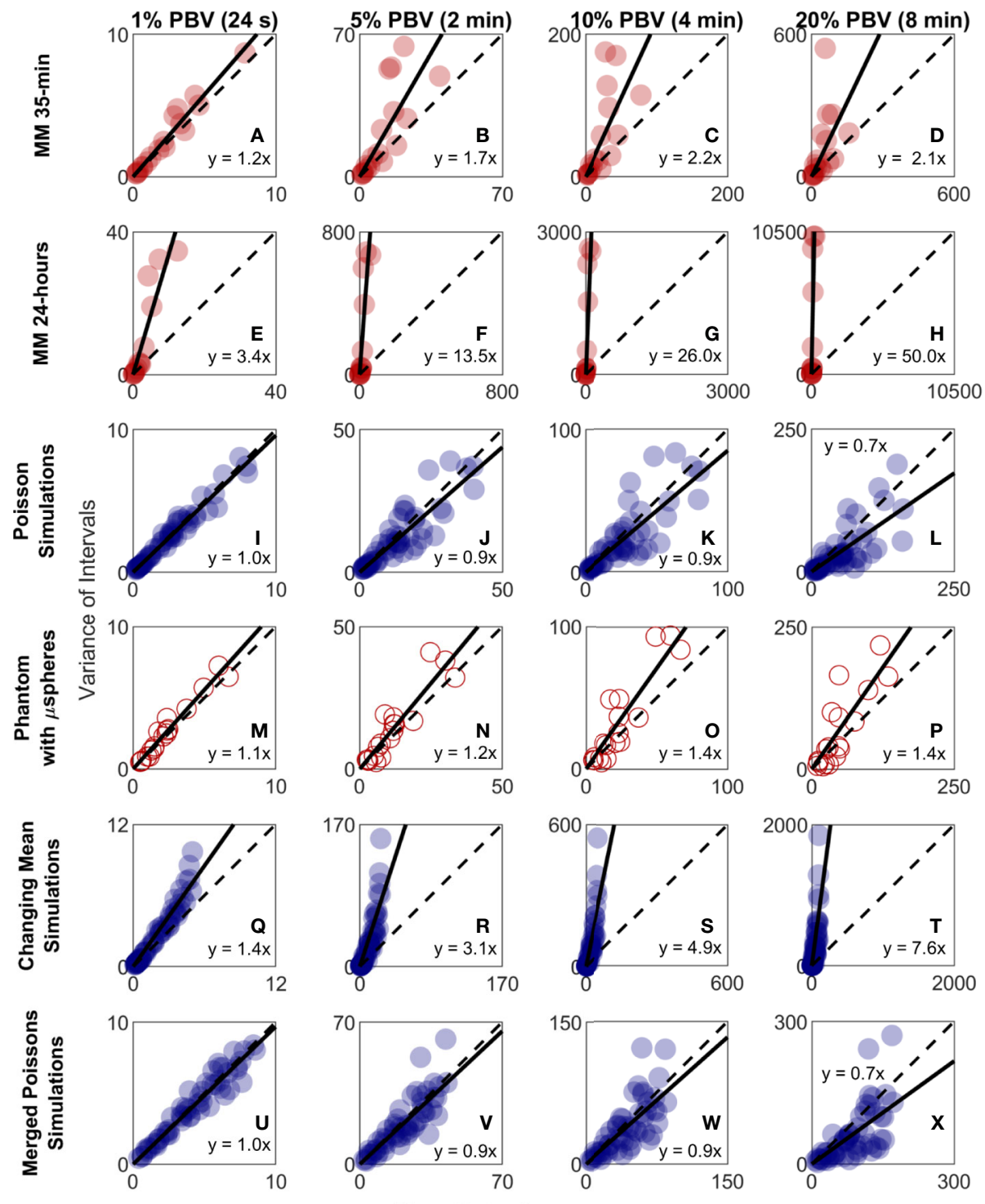

Mean Detections per Interval

FIGURE 6 | The variability in DiFC count rate measured in mice far exceeded that expected by Poisson statistics. Measured variance in CTC counts compared to the scan mean count in DiFC data is shown for (A-D) MM 35-min mice, (E-H) MM 24-h mice, (I-L) simulated Poisson DiFC data, and (M-P) phantoms with suspensions of well-mixed microspheres. The dashed diagonal lines indicate the expected 1:1 variance-to-mean relationship for Poisson-distributed data. (Q-T) Simulations show that the higher than expected variance in MM 35-min data is consistent with a Poisson process with changing mean (see text for details). (U-X) By contrast, two or more simultaneous (merged) Poisson processes would not be consistent with the higher-than-expected variance. Equations and solid lines indicate a linear fit to each data set.

for rare CTCs (LLC bearing mice), any single small sampling interval (equivalent blood sample) frequently resulted in no CTC detections. For more abundant CTCs (MM bearing mice), small samples were unlikely to yield a quantitatively accurate estimate of mean CTC numbers. Data taken over 24-h periods also showed that CTC numbers varied by up to two orders-ofmagnitude, with variance well in excess of that expected by Poisson statistics or operator variability.

In combination, it follows that that CTCs are not well mixed in the PB. This is consistent with two known properties of CTCs, i) that CTCs have a short half-life in circulation, and ii) that CTCs shed continuously from primary tumor(s). For the former, the small number of clinical estimates of CTC half-life range between 6 min and $2.4 \mathrm{~h}(15,30,31)$. Estimates of CTC half-life in mouse models also range from 10 to $60 \mathrm{~min}$ for different cell types and mouse strains $(29,32-37)$. In addition, many CTCs are cleared in minutes in the "first-pass effect" in the lungs, liver, or spleen $(38,39)$. With respect to the kinetics of CTC shedding, it has been previously shown that tumors disseminate CTCs at a rate of about $10^{6}$ cells per gram of tumor tissue per day (40). We 
A

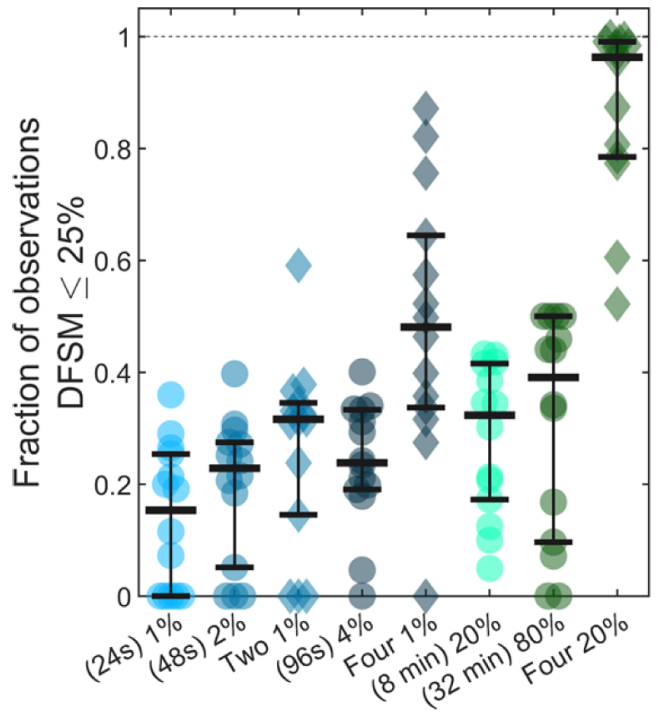

B

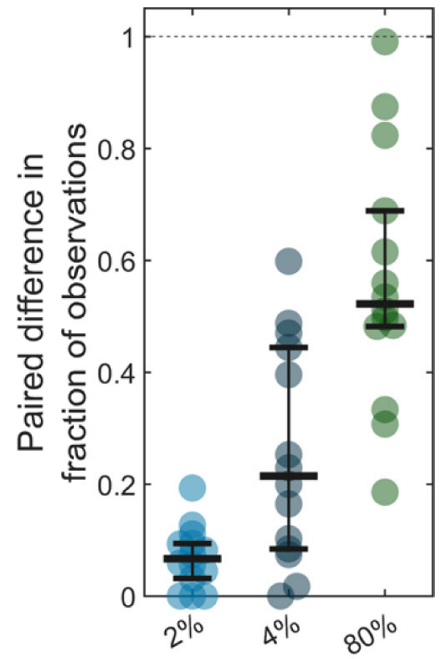

FIGURE 7 | Use of larger blood samples and averaging multiple blood samples yields improved enumeration of CTCs. (A) Fraction of samples of different sizes where the DFSM was less than or equal to $25 \%$ for $1 \%, 2 \%$, two $1 \%, 4 \%$, four $1 \%, 20 \%$, $80 \%$, and four $20 \%$ PBV samples. (B) Paired difference in the fraction of samples were the DFSM <= 25\% between the averaged samples (two 1\%, four $1 \%$, and four $20 \%$ PBV) and a single continuous sample of equivalent size ( $2 \%$, $4 \%$, and $80 \%$ PBV).

were unable to find any specific information on the short-term dynamics of CTC shedding from the primary tumor; however, it is conceivable that this-like other tumor processes-varies over the timescale of minutes. For example, it has been shown that tumors cycle through hypoxia states on similar timescales (41). This suggests that CTCs are continuously and dynamically being shed into and cleared from circulation. For example, in one LLC data set (Figure 3A), only a single detection was observed in a 50 -min DiFC scan suggesting that the CTC cleared from circulation before it could be detected a second time.

DiFC data support the alternate hypothesis that CTC numbers are better described using a kinetic model that oscillates between states of relatively high or low shedding, as opposed to a steady Poisson process. Specifically, CTC data from MM-DXM bearing mice was more consistent with our in silico simulated data sets where the mean CTC rate increased partway through the scan (Figures 6Q-T). These simulations suggested that CTC rates may change by approximately a factor of two over a 35 -min scan, and by larger factors over 24 -h periods. The disseminated nature of the MM tumor also implies that there may be multiple (many) sites of CTC shedding. However, as we noted, multiple simultaneous Poisson-distributed CTC sources would not produce the observed in vivo DiFC data (Figures 6U-X). This suggests that the changing numbers of CTCs in circulation is in response to systemic factors such as hormonal or cardiovascular effects. Despite the similarities in data, our simulations of a doubling in detection rate midway through the scan are, of course, not necessarily representative of what occurs biologically. In general, the magnitude and frequency of CTC rate changes is not known, and moreover is expected to vary with cancer type and mouse strain. Determining an accurate model of these changes is the subject of ongoing work in our group.

Since our observations were made in mouse xenograft models, a natural question is whether similar short-term temporal fluctuations occur in humans. While this requires further study, the general findings here are consistent with the small number of clinical studies in the literature. For example, (22) studied CTC numbers in blood samples taken at 12-h intervals from 51 breast cancer patients. While they concluded that there was no diurnal pattern, blood samples taken on the same day yielded differences by up to a factor of 3.8. Some patients had CTCs detected in one blood sample and no CTCs in the second blood sample, and several switched between prognostic categories (i.e., numbers above or below the 5 CTC threshold). Likewise, (23) showed 24-h variability of up to a factor of 7 in MM patients, and suggested a circadian rhythm to the fluctuations. (42) took three sequential $3 \mathrm{ml} \mathrm{PB}$ samples from each of 7 stage IV melanoma patients, and found also found significant variation in CTC number (by up to a factor of 4), including four patients where no CTCs were detected in some of the samples. However, direct comparison is difficult since these previously reported observations were made using a small number of fractionally small blood samples. As such, they did not permit study of continuous changes in CTC numbers that occur over the timescale of minutes as we do here. Moreover, the limited temporal data did not permit comparison with assumed kinetic or sampling models (such as Poisson statistics).

Together our findings suggest a number of practical implications for the use of liquid biopsy in CTC enumeration. 
As noted the clinical value of CTC numbers as a diagnostic or predictive biomarker is still unclear $(8-10,43,44)$. This has in part driven major efforts toward development of next-generation liquid biopsy technology for characterization of CTC genotypic and phenotypic heterogeneity (12-14). Assuming that the CTC behavior observed here extends to humans, these data may provide a simple complementary explanation (to CTC heterogeneity) for these challenges: CTCs enumerated in fractionally small blood samples is inherently sensitive to the timing of the blood draw because of the natural temporal fluctuations. As shown, these fluctuations could result in change of prognostic category (CTC positive or negative) when simple numerical thresholds are applied.

Second, these data are consistent with the notion that improved clinical CTC enumeration could be achieved by analysis of larger blood samples $(13,14)$, either by liquid biopsy or by development of in vivo methods for counting or capturing CTCs (45). Our analysis suggests that averaging of multiple small blood samples taken over the course of hours should yield even further improved accuracy. Alternatively, new in vivo methods could scan larger blood volumes over time and in principle yield more accurate enumeration of CTCs. In this regard, because DiFC is inherently scalable to larger tissue volumes, we are already exploring the possible translation of DiFC to humans through the use of highly specific fluorescence molecular probes (46).

Third, CTC clusters (CTCCs) are even more rare than CTCs (they occur at a frequency of less than $10 \%$ of single CTCs) but are of great interest because they are known to have significantly higher metastatic potential (47). Although we did not explicitly consider CTCCs in the analysis here, DiFC does permit detection of CTCCs $(27,29)$. The relative rarity of CTCCs implies that the challenges of enumeration with liquid biopsy of small PB samples are likely to be compounded. This is also true when considering accurate enumeration of specific, more-rare CTC phenotypes (11).

Last, the fast turnover of CTCs in PB also suggests that antiCTC therapeutic strategies such as "CTC dialysis" that have been proposed (48-50) are unlikely to succeed unless performed continuously, for example using a wearable device.

In summary, analysis of DiFC data in two mouse models of metastasis shows that CTC numbers are far from steady-state in vivo and undergo significant fluctuations on the timescales of minutes and hours. This can (at least in these models) cause significant error in CTC detection and enumeration using small blood samples and motivates new methods for analyzing larger blood volumes in vivo. Ongoing work by our team includes the application of DiFC to other mouse xenograft models, investigating future clinical use of $\mathrm{DiFC}$, and development of mathematical models to more accurately describe CTC dynamics.

\section{DATA AVAILABILITY STATEMENT}

The datasets generated and analyzed for this study can be found in the Blackfynn repository DOI: $10.26275 / \mathrm{x} 9 \mathrm{xq}-\mathrm{e} 4 \mathrm{wu}$.
Additional data and code used to process data can be found in the Github repository https://github.com/NiedreLab/WilliamsCTC-Dynamics-2020 (DOI: 10.5281/zenodo.4005939).

\section{ETHICS STATEMENT}

The animal study was reviewed and approved by Northeastern University IACUC protocol \#15-0728R.

\section{AUTHOR CONTRIBUTIONS}

AW and $\mathrm{MN}$ designed research. AW, JF, and FI performed research. AW, JF, FI, and ES analyzed data. AW, ES, and MN conceived analysis. AW, ES, and MN wrote the paper. All authors contributed to the article and approved the submitted version.

\section{FUNDING}

This work was supported by the National Institutes of Health (NIH) (R01HL124315; NHLBI).

\section{ACKNOWLEDGMENTS}

We thank Dr. Paul Mathew (Tufts Medical Center, Boston, MA), Prof. Dana Brooks (Northeastern University, Boston, MA), and Prof. Daniel Ocone (Rutgers University, Piscataway, NJ) for their helpful comments and suggestions on our work. We also sincerely thank Dr. Miguel Martin (Universidad Complutense, Madrid, Spain) for kindly sharing data. This manuscript has been released as a pre-print at https://doi.org/10.1101/814368 (51).

\section{SUPPLEMENTARY MATERIAL}

The Supplementary Material for this article can be found online at: https://www.frontiersin.org/articles/10.3389/fonc.2020. 601085/full\#supplementary-material

SUPPLEMENTARY FIGURE 1 | Raster plots of 18 representative DiFC scans from LLC tumor bearing mice ("LLC data set"). Each solid vertical line represents a CTC detection. The dashed lines mark the end of each scan, which were of slightly different lengths. The plots are shown in ascending order of DiFC detection rate.

SUPPLEMENTARY FIGURE 2 | Raster event plots for all 18 DiFC scans in the "MM 35-min date set". Each vertical line represents a CTC detection. The plots are in ascending order of $\mathrm{DiFC}$ detection rate.

SUPPLEMENTARY FIGURE 3 | Fluctuations in the mean CTC count rate over 24-h periods for all DiFC scans the "MM 24-h data set". Triangle markers identify the first scan of the 24-h cycle. $\mathrm{N}=7$ cycles started $\sim 0700$ ( $7 \mathrm{am})$ and $\mathrm{N}=7$ sessions began at 1900 (7 pm). Animal housing followed a 0700 to 1900 light (white background) and 1900 and 0700 dark (gray background) light cycles. 


\section{REFERENCES}

1. Gupta GP, Massague J. Cancer metastasis: building a framework. Cell (2006) 127(4):679-95. doi: 10.1016/j.cell.2006.11.001

2. Steeg PS, Theodorescu D. Metastasis: a therapeutic target for cancer. Nat Clin Pract Oncol (2008) 5(4):206-19. doi: 10.1038/ncponc1066

3. Alix-Panabieres C, Pantel K. Circulating tumor cells: liquid biopsy of cancer. Clin Chem (2013) 59(1):110-8. doi: 10.1373/clinchem.2012.194258

4. Mader S, Pantel K. Liquid biopsy: current status and future perspectives. Oncol Res Treat (2017) 40(7-8):404-8. doi: 10.1159/000478018

5. Cristofanilli M, Budd GT, Ellis MJ, Stopeck A, Matera J, Miller MC, et al. Circulating tumor cells, disease progression, and survival in metastatic breast cancer. N Engl J Med (2004) 351(8):781-91. doi: 10.1056/NEJMoa040766

6. Cohen SJ, Punt CJ, Iannotti N, Saidman BH, Sabbath KD, Gabrail NY, et al. Relationship of circulating tumor cells to tumor response, progression-free survival, and overall survival in patients with metastatic colorectal cancer. J Clin Oncol (2008) 26(19):3213-21. doi: 10.1200/JCO.2007.15.8923

7. Moreno JG, Miller MC, Gross S, Allard WJ, Gomella LG, Terstappen LW. Circulating tumor cells predict survival in patients with metastatic prostate cancer. Urology (2005) 65(4):713-8. doi: 10.1016/j.urology.2004.11.006

8. Smerage JB, Barlow WE, Hortobagyi GN, Winer EP, Leyland-Jones B, Srkalovic G, et al. Circulating tumor cells and response to chemotherapy in metastatic breast cancer: SWOG S0500. J Clin Oncol (2014) 32(31):3483-9. doi: 10.1200/JCO.2014.56.2561

9. Raimondi C, Gradilone A, Naso G, Cortesi E, Gazzaniga P. Clinical utility of circulating tumor cell counting through CellSearch $((\mathrm{R}))$ : the dilemma of a concept suspended in Limbo. Onco Targets Ther (2014) 7:619-25. doi: 10.2147/OTT.S46200

10. Alix-Panabieres C, Pantel K. Challenges in circulating tumour cell research. Nat Rev Cancer (2014) 14(9):623-31. doi: 10.1038/nrc3820

11. Polzer B, Medoro G, Pasch S, Fontana F, Zorzino L, Pestka A, et al. Molecular profiling of single circulating tumor cells with diagnostic intention. EMBO Mol Med (2014) 6(11):1371-86. doi: 10.15252/emmm.201404033

12. Bardia A, Haber DA. Solidifying liquid biopsies: can circulating tumor cell monitoring guide treatment selection in breast cancer? J Clin Oncol (2014) 32 (31):3470-1. doi: 10.1200/JCO.2014.57.1505

13. Allan AL, Keeney M. Circulating tumor cell analysis: technical and statistical considerations for application to the clinic. J Oncol (2010) 2010:426218. doi: $10.1155 / 2010 / 426218$

14. Lalmahomed ZS, Kraan J, Gratama JW, Mostert B, Sleijfer S, Verhoef C. Circulating tumor cells and sample size: the more, the better. J Clin Oncol (2010) 28(17):e288-9; author reply e90. doi: 10.1200/JCO.2010.28.2764

15. Stott SL, Lee RJ, Nagrath S, Yu M, Miyamoto DT, Ulkus L, et al. Isolation and characterization of circulating tumor cells from patients with localized and metastatic prostate cancer. Sci Transl Med (2010) 2(25):25ra3. doi: 10.1126/ scitranslmed.3000403

16. Karabacak NM, Spuhler PS, Fachin F, Lim EJ, Pai V, Ozkumur E, et al. Microfluidic, marker-free isolation of circulating tumor cells from blood samples. Nat Protoc (2014) 9(3):694-710. doi: 10.1038/nprot.2014.044

17. Au SH, Edd J, Stoddard AE, Wong KHK, Fachin F, Maheswaran S, et al. Microfluidic Isolation of Circulating Tumor Cell Clusters by Size and Asymmetry. Sci Rep (2017) 7(1):2433. doi: 10.1038/s41598-017-01150-3

18. Hoff J. Methods of blood collection in the mouse. Lab Anim (2000) 29(10):47-53.

19. Hartmann C, Patil R, Lin CP, Niedre M. Fluorescence detection, enumeration and characterization of single circulating cells in vivo: technology, applications and future prospects. Phys Med Biol (2017) 63(1):01TR. doi: 10.1088/13616560/aa98f9

20. Tibbe AG, Miller MC, Terstappen LW. Statistical considerations for enumeration of circulating tumor cells. Cytometry A (2007) 71(3):154-62. doi: 10.1002/cyto.a.20369

21. Coumans FA, Ligthart ST, Terstappen LW. Interpretation of changes in circulating tumor cell counts. Transl Oncol (2012) 5(6):486-91. doi: 10.1593/ tlo. 12247

22. Martin M, Garcia-Saenz JA, Maestro De las Casas ML, Vidaurreta M, Puente J, Veganzones S, et al. Circulating tumor cells in metastatic breast cancer: timing of blood extraction for analysis. Anticancer Res (2009) 29(10):4185-7.

23. Paiva B, Paino T, Sayagues JM, Garayoa M, San-Segundo L, Martin M, et al. Detailed characterization of multiple myeloma circulating tumor cells shows unique phenotypic, cytogenetic, functional, and circadian distribution profile. Blood (2013) 122(22):3591-8. doi: 10.1182/blood-2013-06-510453

24. Tuchin VV, Tarnok A, Zharov VP. In vivo flow cytometry: a horizon of opportunities. Cytometry A (2011) 79(10):737-45. doi: 10.1002/cyto.a.21143

25. Zettergren E, Vickers D, Runnels J, Murthy SK, Lin CP, Niedre M. Instrument for fluorescence sensing of circulating cells with diffuse light in mice in vivo. J BioMed Opt (2012) 17(3):37001. doi: 10.1117/1.JBO.17.3.037001

26. Tan $X$, Patil R, Bartosik P, Runnels JM, Lin CP, Niedre M. In vivo flow cytometry of extremely rare circulating cells. Sci Rep (2019) 9(1):3366. doi: 10.1038/s41598-019-40143-2

27. Patil R, Tan X, Bartosik P, Detappe A, Runnels JM, Ghobrial I, et al. Fluorescence monitoring of rare circulating tumor cell and cluster dissemination in a multiple myeloma xenograft model in vivo. J BioMed Opt (2019) 24(8):1-11. doi: 10.1117/1.JBO.24.8.085004

28. Di W, Tan X, Calderon IAC, Neal Reilly AE, Niedre M, Clark HA. Real-time particle-by-particle detection of erythrocyte-camouflaged microsensor with extended circulation time in the bloodstream. Proc Natl Acad Sci U S A (2020) 117(7):3509-17. doi: 10.1073/pnas.1914913117

29. Fitzgerald JE, Byrd BK, Patil RA, Strawbridge RR, Davis SC, Bellini C, et al. Heterogeneity of circulating tumor cell dissemination and lung metastases in a subcutaneous Lewis lung carcinoma model. BioMed Opt Express (2020) 11 (7):3633-47. doi: 10.1364/BOE.395289

30. Meng S, Tripathy D, Frenkel EP, Shete S, Naftalis EZ, Huth JF, et al. Circulating tumor cells in patients with breast cancer dormancy. Clin Cancer Res (2004) 10(24):8152-62. doi: 10.1158/1078-0432.CCR-041110

31. Aceto N, Bardia A, Miyamoto DT, Donaldson MC, Wittner BS, Spencer JA, et al. Circulating tumor cell clusters are oligoclonal precursors of breast cancer metastasis. Cell (2014) 158(5):1110-22. doi: 10.1016/ j.cell.2014.07.013

32. Georgakoudi I, Solban N, Novak J, Rice WL, Wei X, Hasan T, et al. In vivo flow cytometry: a new method for enumerating circulating cancer cells. Cancer Res (2004) 64(15):5044-7. doi: 10.1158/0008-5472.CAN-04-1058

33. Sipkins DA, Wei X, Wu JW, Runnels JM, Cote D, Means TK, et al. In vivo imaging of specialized bone marrow endothelial microdomains for tumour engraftment. Nature (2005) 435(7044):969-73. doi: 10.1038/nature03703

34. He W, Wang H, Hartmann LC, Cheng JX, Low PS. In vivo quantitation of rare circulating tumor cells by multiphoton intravital flow cytometry. Proc Natl Acad Sci U S A (2007) 104(28):11760-5. doi: 10.1073/pnas.0703875104

35. Runnels JM, Carlson AL, Pitsillides C, Thompson B, Wu J, Spencer JA, et al. Optical techniques for tracking multiple myeloma engraftment, growth, and response to therapy. J BioMed Opt (2011) 16(1):011006. doi: 10.1117/ 1.3520571

36. Pestana N, Mortensen LJ, Runnels JP, Vickers D, Murthy SK, Lin CP, et al. Improved diffuse fluorescence flow cytometer prototype for high sensitivity detection of rare circulating cells in vivo. J BioMed Opt (2013) 18(7):77002. doi: 10.1117/1.JBO.18.7.077002

37. Pera V, Tan X, Runnels J, Sardesai N, Lin CP, Niedre M. Diffuse fluorescence fiber probe for in vivo detection of circulating cells. J BioMed Opt (2017) 22 (3):37004. doi: 10.1117/1.JBO.22.3.037004

38. Mizuno N, Kato Y, Izumi Y, Irimura T, Sugiyama Y. Importance of hepatic first-pass removal in metastasis of colon carcinoma cells. J Hepatol (1998) 28 (5):865-77. doi: 10.1016/s0168-8278(98)80238-9

39. Fischer UM, Harting MT, Jimenez F, Monzon-Posadas WO, Xue H, Savitz SI, et al. Pulmonary passage is a major obstacle for intravenous stem cell delivery: the pulmonary first-pass effect. Stem Cells Dev (2009) 18(5):683-92. doi: $10.1089 /$ scd.2008.0253

40. Butler TP, Gullino PM. Quantitation of cell shedding into efferent blood of mammary adenocarcinoma. Cancer Res (1975) 35(3):512-6.

41. Dewhirst MW. Relationships between cycling hypoxia, HIF-1, angiogenesis and oxidative stress. Radiat Res (2009) 172(6):653-65. doi: 10.1667/RR1926.1

42. Juratli MA, Sarimollaoglu M, Nedosekin DA, Melerzanov AV, Zharov VP, Galanzha EI. Dynamic fluctuation of circulating tumor cells during cancer progression. Cancers (Basel) (2014) 6(1):128-42. doi: 10.3390/cancers6010128

43. de Bono JS, Scher HI, Montgomery RB, Parker C, Miller MC, Tissing H, et al. Circulating tumor cells predict survival benefit from treatment in metastatic castration-resistant prostate cancer. Clin Cancer Res (2008) 14(19):6302-9. doi: 10.1158/1078-0432.CCR-08-0872 
44. Toss A, Mu Z, Fernandez S, Cristofanilli M. CTC enumeration and characterization: moving toward personalized medicine. Ann Transl Med (2014) 2(11):108. doi: 10.3978/j.issn.2305-5839.2014.09.06

45. Saucedo-Zeni N, Mewes S, Niestroj R, Gasiorowski L, Murawa D, Nowaczyk P, et al. A novel method for the in vivo isolation of circulating tumor cells from peripheral blood of cancer patients using a functionalized and structured medical wire. Int J Oncol (2012) 41(4):1241-50. doi: 10.3892/ijo.2012.1557

46. Patil RA, Srinivasarao M, Amiji MM, Low PS, Niedre M. Fluorescence labeling of circulating tumor cells with a folate receptor-targeted molecular probe for diffuse In vivo flow cytometry. Mol Imaging Biol (2020) 22:1280-9. doi: 10.1007/s11307-020-01505-9

47. Hong Y, Fang F, Zhang Q. Circulating tumor cell clusters: What we know and what we expect (Review). Int J Oncol (2016) 49(6):2206-16. doi: 10.3892/ ijo.2016.3747

48. Faltas B. Cornering metastases: therapeutic targeting of circulating tumor cells and stem cells. Front Oncol (2012) 2:68. doi: 10.3389/fonc.2012.00068

49. Kim YR, Yoo JK, Jeong CW, Choi JW. Selective killing of circulating tumor cells prevents metastasis and extends survival. J Hematol Oncol (2018) 11 (1):114. doi: 10.1186/s13045-018-0658-5
50. Galanzha EI, Menyaev YA, Yadem AC, Sarimollaoglu M, Juratli MA, Nedosekin DA, et al. In vivo liquid biopsy using Cytophone platform for photoacoustic detection of circulating tumor cells in patients with melanoma. Sci Transl Med (2019) 11(496):eaat5857. doi: 10.1126/scitranslmed.aat5857

51. Williams AL, Fitzgerald JE, Ivich F, Sontag ED, Niedre M. Short-term circulating tumor cell dynamics in mouse xenograft models and implications for liquid biopsy. bioRxiv (2020) Preprint:814368. doi: 10.1101/ 814368

Conflict of Interest: The authors declare that the research was conducted in the absence of any commercial or financial relationships that could be construed as a potential conflict of interest.

Copyright (c) 2020 Williams, Fitzgerald, Ivich, Sontag and Niedre. This is an open-access article distributed under the terms of the Creative Commons Attribution License (CC BY). The use, distribution or reproduction in other forums is permitted, provided the original author(s) and the copyright owner(s) are credited and that the original publication in this journal is cited, in accordance with accepted academic practice. No use, distribution or reproduction is permitted which does not comply with these terms. 